Communications in Physics, Vol.25, No. 1 (2015), pp. 59-65

DOI:10.15625/0868-3166/25/1/0

\title{
DEVELOPMENT OF A FLUORESCENCE CORRELATION SPECTROSCOPY INSTRUMENT AND ITS APPLICATION IN SIZING QUANTUM DOT NANOPARTICLES
}

\author{
NGUYEN THI THANH BAO AND DINH VAN TRUNG \\ Institute of Physics, Vietnam Academy of Science and Technology \\ 10 DaoTan, BaDinh, Hanoi, Vietnam
}

E-mail: ntbao@iop.vast.ac.vn

Received 08 December 2014

Accepted for publication 06 March 2015

\begin{abstract}
Fluorescence correlation spectroscopy is a relatively new technique to measure and quantify the statistical fluctuations of the fluorescence signal from the measurement volume. Combining with sensitive detection method and confocal microscopy, the FCS technique has become a powerful tool in studying the dynamic properties of nanoparticles at single particle level. In this paper we present the construction of a highly sensitive FCS instrument and the measurement results from a sample of semiconductor quantum dots. We provide the analysis procedure for determining the hydrodynamic radius of the quantum dots and compare the results with the ones obtained directly from electron microscope imaging. The good agreement indicates the reliability of the FCS technique and open the way for further applications of this technique in studying nanoparticles.
\end{abstract}

Keywords: FCS; quantum dots; hydrodynamic radius; photon counting..

\section{INTRODUCTION}

Characterization of nanoparticles has recently found wide applications in several important research fields such as nanotechnology, biophysics, chemistry etc... In many instances, the nanoparticles being studied exist in liquid medium such as solution or intra-cellular environment. Direct measurement of freely diffusing nanoparticles in these cases provides a convenient and noninvasive way to obtain data in real-time, where other characterization methods such as direct imaging are unsuitable. In this aspect, the fluorescence correlation spectroscopy (FCS) technique has become the method of choice for a number of problems related to the diffusion dynamics of nanoparticle or single molecules in liquid environments [1]. The FCS technique was first introduced by Magde et al. (1972) [2] to study the dynamics within a reacting system. The full potential of this technique was later realized with the introduction of confocal microscopy excitation and detection configuration, yielding small detection volume, and the use of highly sensitive single photon detectors by Rigler et al. (1993) [3]. The detection volume is created by tightly focusing 
60DEVELOPMENT OF A FLUORESCENCE CORRELATION SPECTROSCOPY INSTRUMENT AND ITS APPLICATION ...

of excitation laser beam into the solution sample and by using a confocal pinhole in the detection pathway. The detection volume then becomes very small, of the order of femto-litter, from which the fluorescence light is collected, leading to a very little amount of sample required for analysis. As a result, one can follow and determine the rates of movement of extremely small species such as molecules or nanoscale particles [1, 4-8]. The high sensitivity of the FCS technique allows measurements of fluorescent molecules or nano particles at very low concentration, in the range pM to nM. Currently, the theoretical basis for FCS measurement has been well established, allowing a very detailed analysis of the measurement data to derive accurately the dynamic properties of nanoparticles.

In this paper we provide the general description of the FCS technique together with detailed information on the construction of the first FCS instrument in Vietnam. The characterization of our instrument is carried out using dye molecules with known diffusion properties and, in that process, demonstrating the high sensitivity, up to single molecule level, of the instrument. Finally, we apply the FCS technique to measure the dynamics and the average hydrodynamic size of quantum dots samples.

\section{THEORETICAL BASIS OF FCS}

In a FCS experiment, individual fluorescent photons originating from the small detection volume, typically of femto-liter size within the solution sample, are detected. The number of such photons $I(t)$ within a certain time bin, or the fluorescent intensity, are then counted as a function of time. Due to the Brownian motion, the nanoparticles diffuse freely into and out of the detection volume. As the detection volume is in the range of femto-liter, on average only a small number of nanoparticles are present in the detection volume at any time. The diffusion of a single nanoparticle into or out of the dection volume leads to a large change in the measured fluorescent intensity $I(t)$. The change or fluctuation of $I(t)$ carries the information about the diffusion dynamics, i.e. the diffusion coefficient or the rate constants of chemical reactions, of the system under consideration [1-4].

The diffusion of nanoparticles in solution is well characterized by the diffusion constant $D$, which is related to the size of the nanoparticle and the thermodynamic properties of the solution through the Stokes-Einstein relation:

$$
D=\frac{k T}{6 \pi \eta R}
$$

where $T$ is the temperature, $\eta$ is the solvent viscosity and $R$ is the hydrodynamic radius of the nanoparticles.

The normalized autocorrelation function, $G(\tau)$, of the fluorescence fluctuations is calculated from the fluorescence intensity $I(t)$ as follows:

$$
G(\tau)=\frac{\langle\delta I(t) \delta I(t+\tau)\rangle}{\langle I(t)\rangle^{2}}
$$

The fluctuations are calculated as $\delta I(t)=I(t)-\langle I(t)\rangle$ and the brackets $\langle\ldots\rangle$ denote time average. The temporal decay of $G(\tau)$ contains information on the timescale of the dynamic processes causing the fluorescence fluctuations. It was well established that the autocorrelation function $G(\tau)$ is 
related to the diffusion time and the characteristics of the FCS setup:

$$
G(\tau)=\frac{1}{N}\left[1+\frac{\tau}{\tau_{D}}\right]^{-1}\left[1+\frac{\tau}{\tau_{D}}\left(\frac{r_{0}}{z_{o}}\right)^{2}\right]^{-1 / 2}
$$

The detection volume is assumed to have a three-dimensional Gaussian shape with radial and axial size of $r_{0}$ and $z_{0}$ over which the fluorescent intensity decays spatially by $1 / e$. Here, $N$ is the mean number of nanoparticles in the effective detection volume $N=c V_{\text {eff }}, c$ is concentration of nanoparticles, $V_{\text {eff }}=\pi^{3 / 2} r_{0}^{2} z_{0}$. The time constant $\tau_{D}$ is the correlation time for translational diffusion and related to the diffusion constant $\mathrm{D}$ defined above as:

$$
\tau_{D}=\frac{r_{0}^{2}}{4 D}
$$

The parameters $r_{0}$ and $z_{0}$ are the main characteristics of the FCS setup and can be calibrated by measuring the diffusion time $\tau_{D}$ of known samples of particles, usually dye molecules, with known diffusion constant D. By using nonlinear least-square fitting of the autocorrelation function $G(\tau)$, the diffusion time $\tau_{D}$ and the ratio $r_{0} / z_{0}$ could be determined, allowing us to estimate the real value of $r_{0}$ and $z_{0}$.

For nanoparticles under study, one can derive from fitting the autocorrelation function the diffusion time $\tau_{D}$, which in turn allows the estimation of the diffusion constant $D$. Using the Stokes-Einstein relation, one can calculate the value of the hydrodynamic radius of the nanoparticle, with the knowledge of the temperature and the viscosity of the solvent.

\section{EXPERIMENT SETUP}

We have setup a FCS instrument in our laboratory at the Institute of Physics. The schematics of the setup is shown in Fig. 1. The excitation laser is a $\mathrm{Nd}$ :YAG laser with an average power of $50 \mathrm{~mW}$. The laser is temperature controlled using a Peltier element. A set of neutral density filters is used to reduce the laser intensity to an appropriate level for measurement. A spatial filter consisting of a pair of lenses L1 and L2 and a $20 \mu \mathrm{m}$ pinhole is used to clean up the laser beam to provide a pure $\mathrm{TEM}_{00}$ mode. This is very important to obtain diffraction - limited

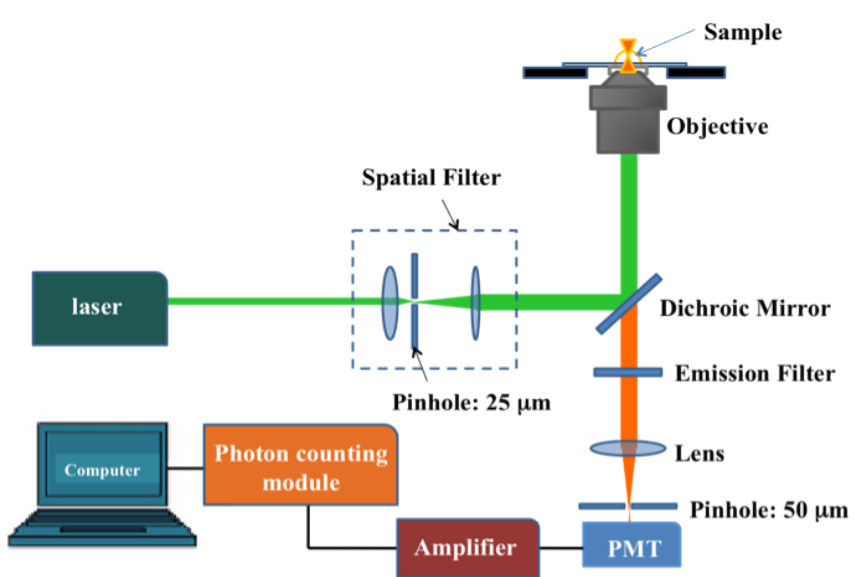

Fig. 1. Schematic diagram of the FCS instrument. excitation volume in the sample. The laser beam is then passed through the aperture A and directed by a dichroic mirror DM toward a high numerical aperture oil immersion microscope objective (Edmund Optics, NA $=1.25$ ). The laser beam is focused by this microscope objective into the sample prepared on a thin $(0.17 \mathrm{~mm}$ 
in thickness) microscope coverslip. The fluorescent light from the sample is then collected by the same objective, transmitted through the dichroic mirror DM and reflected by mirror M2 and focused by a lens $\mathrm{L} 3$ of $15 \mathrm{~cm}$ in focal length onto the $50 \mu \mathrm{m}$ confocal pinhole. A combination of an interference longpass filter and bandpass filter placed right behind the confocal pinhole block the scattered laser light and any background light while passing the fluorescent light. Then the fluorescent light is detected by two photomultiplier tubes of the compact head-on type R9880 (Hamamatsu, Japan) operating in photon counting mode. The fluorescence photons detected in each channel are first amplified by a high speed amplifier developed by our group and then counted to form the correlation function using Picoharp 300 module from Picoquant.

In our measurements, the samples of dye molecules or quantum dots are prepared in water with predetermined concentration and transferred to the thin cover slip of $0.13-0.17 \mathrm{~mm}$ in thickness using micropipettes. Laser grade dye Rhodamine B is used for calibration purpose of the FCS instrument. CdTe/CdS quantum dots fabricated at the Institute of Material Science are used.

To adjust the excitation laser power to suitable levels, we use different neutral density filters ranging from $\mathrm{OD}=0.5$ to 2 before the spatial filter. The laser power after the spatial filter is measured using a Newport power meter and shown to be in the range from 3 to $400 \mu \mathrm{W}$.

\section{CALIBRATION OF THE FCS INSTRUMENT}

The first step in any FCS measurement is to accurately characterize the properties of the instrument. We use the standard procedure of measuring the correlation function and the diffusion time of a dye molecule with known diffusion coefficient. We choose to use a solution of Rhodamine $\mathrm{B}$ dye at extremely dilute concentration in water. The Rhodamine B dye molecules when excited by green light at $532 \mathrm{~nm} \mathrm{Nd:YAG} \mathrm{laser} \mathrm{pro-}$ duce strong fluorescence in the yellow red wavelength region, peaking around $580 \mathrm{~nm}$. With the optimal setup for laser excitation volume, we successfully obtain the autocorrelation curve for Rhodamine B in 5 to 10 minutes as shown in Fig. 2. By performing a non-linear least square fitting of the measurement data to the theoretical autocorrelation function $G(\tau)$ we obtain the value of $G(0)=0.6, \tau_{D}=38 \mu$ s and the geometric ratio $r_{0} / z_{0}=0.11$, respectively. Because the diffusion

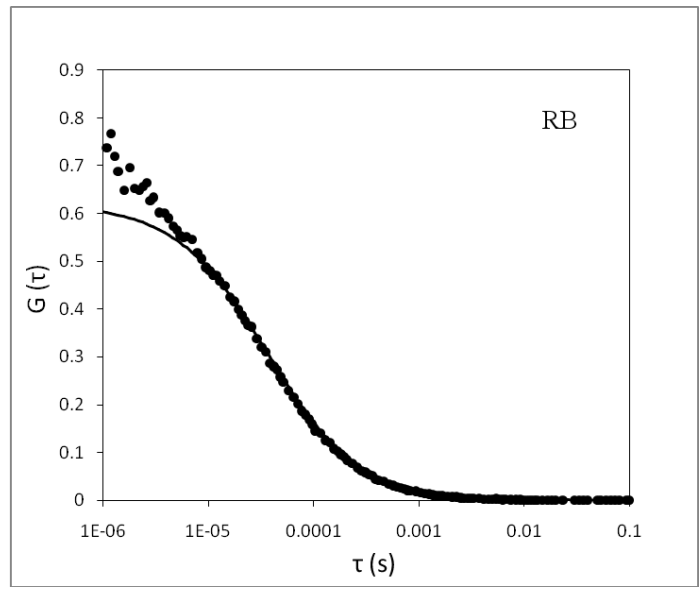

Fig. 2. The autocorrelation measurement (filled circles) of Rhodamine B dye molecules in water. The thin solid line represents the theoretical fit to the measurement data. coefficient $\mathrm{D}$ of Rhodamine $\mathrm{B}$ is well known to be $4.5 \times 10^{-6} \mathrm{~cm}^{2} \mathrm{~s}^{-1}$ [8], we can easily deduce from Eq. (1) the radius of the observation volume $r_{0}=260 \mathrm{~nm}$. That leads to $z_{0}=2.37 \mu \mathrm{m}$ and the effective volume $V_{\text {eff }}=0.89 \mathrm{fl}$. The amplitude $G(0)$ of the correlation curve gives the number of molecules, $N=1.7$, in the detection volume, corresponding to an average concentration of $3.2 \mathrm{nM}$. This measurement showed that we indeed have reached the single molecule level with our FCS instrument. Samples of other fluorescent molecules or nanoparticles can now be measured using this well-defined observation geometry. 
The changes in objective position with respect to the sample are minimized and the geometry characteristics are held fixed in the analysis.

\section{QUANTUM DOT SIZE DETERMINATION}

With the instrument fully calibrated, we then carried out the fluorescence correlation measurements of quantum dot samples. The quantum dots we used emit a wide fluorescent spectrum in the yellow red spectral region, peaking at wavelength of $574 \mathrm{~nm}$. Our FCS instrument collects the emission in the wavelength region redward of $575 \mathrm{~nm}$. An interesting effect in FCS measurement is the dependence of the autocorrelation data on the excitation intensity. This effect is particularly evident in the case of quantum dots as we show in Fig. 3 the autocorrelation data obtained at different power level of the excitation laser radiation. We choose to carry out our FCS measurements with a laser power of $30 \mu \mathrm{W}$, for which the influence of laser power level is minimal on the measurements and the signal to noise ratio is also sufficient for reliable data analysis.

The measured auto-correlation data are shown in Fig. 4. By fitting the theoretical auto-correlation function $G(\tau)$ to the measured data, we obtain a diffusion time $\tau=160 \mu \mathrm{s}$. The much larger diffusion time of quantum dots is a clear demonstration of their large size and thus quantum dots diffuse at significantly lower speed in solution in comparison to dye molecules. Using the parameters of our FCS instrument determined from the calibration procedure described above, we derive a diffusion constant $D=1.06 \times 10^{-6} \mathrm{~cm}^{2} \mathrm{~s}^{-1}$ for the sam-

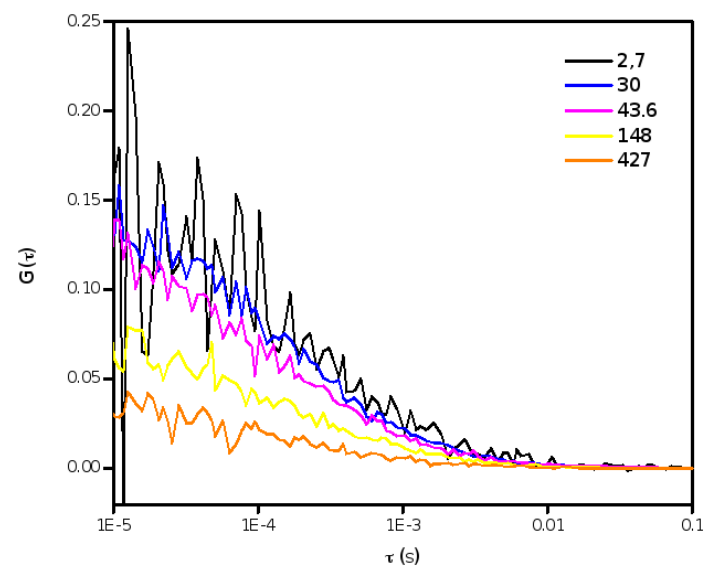

Fig. 3. Autocorrelation measurements of quantum dots at different excitation laser power in $\mu \mathrm{W}$.

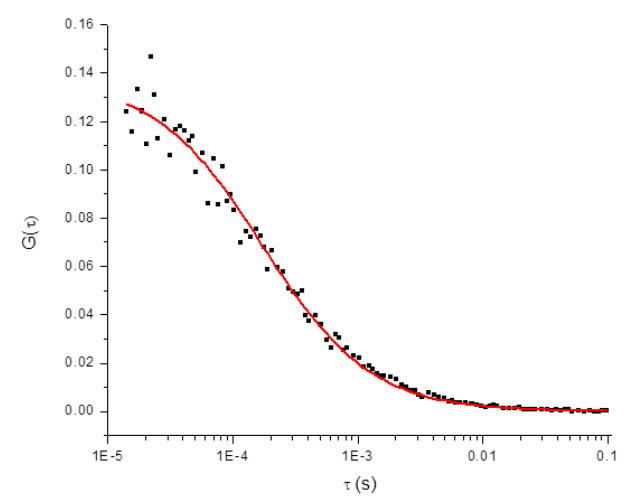

Fig. 4. Autocorrelation data for quantum dots (filled squares) obtained at laser power of $30 \mu \mathrm{W}$ and the theoretical fit (solid line) of the autocorrelation function $G(\tau)$ to the data.

ple of $\mathrm{CdTe} / \mathrm{CdS}$ quantum dots.

Applying the Stokes-Einstein relation expressed in Eq. (1), using known values of viscosity of water $\eta=8.9 \times 10^{-4}$ Pa.s [8], and the room temperature $T=298 \mathrm{~K}$, we obtain an average hydrodynamic size of approximately $4.6 \mathrm{~nm}$ for the quantum dots. The error in the size measurement is mainly related to the calibration of the FCS instrument and the statistical fluctuation of the autocorrelation data. We estimate that the error of the average hydrodynamic size is less than 
$10 \%$. To better understand the accuracy of the size determination, we compare the hydrodynamic size obtained from FCS with that determined from a direct approach. Direct determination of the size of quantum dots in our study is carried out using the TEM image obtained with transmission electron microscope model JEOL-JEM 1010 showed in Fig. 5. However, due to the small size of the quantum dots in our sample and the resolution of the TEM instrument, the TEM image does not allow an accurate estimate of the size distribution. The size of a few quantum dots calculated directly from the imaging data is also shown in Fig. 5 and ranges from 3.5 to $4.1 \mathrm{~nm}$. Evidently, the hydrodynamic size is consistent and in agreement with that determined from FCS measurement. The result indicates a good performance and reliability of our FCS instrument. Furthermore, we notice that the hydrodynamic size is slightly larger than the geometric size of the quantum dots as determined from TEM imaging.

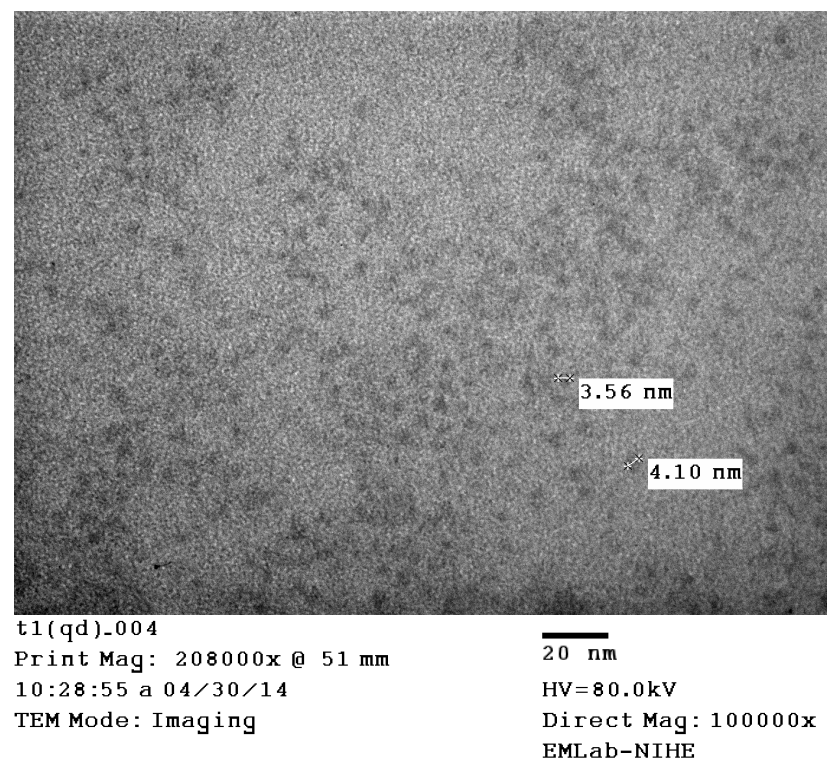

Fig. 5. TEM image of the CdTe/CdS quantum dots used in our measurement. The size of the quantum dots is approximately $4 \mathrm{~nm}$.

Such phenomenon is commonly observed and is mainly due to the fact that in water solution the quantum dot nanoparticles interact with the surrounding, leading to the attachment of water molecules to the quantum dots and to the formation of a surface layer. The presence of a surface layer leads to an effective increase of the measured particle size [9].

\section{CONCLUSION}

In this paper we have presented the details of our FCS instrument developed at the Institute of Physics. The single molecule sensitivity of our instrument has been demonstrated, allowing us to begin the study of single molecule and single particle level. The calibration procedure for the instrument is also established to fully characterize the properties of the setup. One of the applications of FCS technique is to measure the hydrodynamic size: the quantum dots synthesized 
locally show broad agreement between hydrodynamic size and the directly measured geometric size.

\section{ACKNOWLEDGEMENTS}

The financial support from Vietnam Academy of Science and Technology to the authors through projects VAST02.03/11-12 and VAST01.01/12-13 is greatly acknowledged. This research has made use of NASA's Astrophysics Data System.

\section{REFERENCES}

[1] E. Haustein, P. Schwille, Methods 29 (2003) 153 - 166.

[2] D. Magde, E.L. Elson, W.W. Webb, Phys. Rev. Lett. 29 (1972) 705 - 708.

[3] R. Rigler, U. Mets, J. Widengren, P. Kask, Eur. Biophys. J. 22 (1993) 169 - 175.

[4] R. F. Heuff, J. L. Swift, D. T. Cramb, Phys. Chem. Chem. Phys. 9 (2007) $1870-1880$.

[5] C. L. Kuyper, B. S. Fujimoto, Y. Q. Zhao, P. G. Schiro, D. T. Chiu, J. Phys. Chem. 110 (2006) 24433 - 24441.

[6] R. Rieger, C. Rocker, G. Ulrich Nienhaus, Am. J. Phys. 73 (2005) 1129 - 1134.

[7] S.H. Kim, T. Shim, D. Kim, B. Zhong Tang, J. Korean Phys. Soc. 56 (2010) $1264-1268$

[8] P. Kapusta, Picoquant Application note (2001). http://www.picoquant.com/images/uploads/page/ files/7353/appnote_diffusioncoefficients.pdf

[9] P. Zhang, L. Li, C. Q. Dong, H. F. Qian, J. C. Ren, Anal. Chim. Acta 546 (2005) 46 - 51. 